\title{
Bibliometric analysis of the automatic indexing literature: 1956-2000
}

\author{
Antonio Pulgarín a, Isidoro Gil-Leiva ${ }^{\text {b }}$ \\ ${ }^{a}$ Faculty of Library \& Information Science, University of Extremadura, La Alcazaba, 06071 Badajoz, Spain \\ ${ }^{b}$ Faculty of Computer Sciences, Polytechnic University of Valencia, Spain
}

\begin{abstract}
We present a bibliometric study of a corpus of 839 bibliographic references about automatic indexing, covering the period 1956-2000. We analyse the distribution of authors and works, the obsolescence and its dispersion, and the distribution of the literature by topic, year, and source type. We conclude that: (i) there has been a constant interest on the part of researchers; (ii) the most studied topics were the techniques and methods employed and the general aspects of automatic indexing; (iii) the productivity of the authors does fit a Lotka distribution (Dmax = 0.02 and critical value $=0.054$ ); (iv) the annual aging factor is $95 \%$; and (v) the dispersion of the literature is low.
\end{abstract}

Keywords: Automatic indexing; Scientific output; Bibliometric analysis

${ }^{a}$ Corresponding author. Tel. : +34- 924-286406; fax: +34-924-260680

E-mail address: apulgue@alcazaba.unex.es (A. Pulgarín), isgil@har.upv.es (I. Gil-Leiva). 


\section{Introduction}

Indexing, the procedure applied to documents and queries to select their essential concepts, has the function of allowing the storage in databases as well as the later retrieval (two sides of the same coin).

As indexing is an intellectual process (reading, comprehension, analysis, representation), one of its characteristics is having a strong subjective component, and indeed it can be said that subjectivity is inherent to indexing. One way to detect the subjectivity in indexing is to study its consistency, either between different indexers in their analysis of the same document, or between analyses of the same document by the same indexer at different times. The complexity of indexing is very clearly presented in the recent reviews (Anderson \& Pérez-Carballo, 2001a, b), and this difficulty becomes even greater when the aim is to make indexing automatic.

At the beginning of the 1970s, a debate began about whether or not automating the process of indexing was worthwhile. Apart from this debate, the process has aroused clear interest amongst researchers, given the quantity of literature produced in the last half century.

This quantity of research literature has led to the appearance of new techniques for both the treatment of the information and its later retrieval. The changes took place thanks to the first generally available computers which made it possible to carry out rapid repetitive mechanical operations, and, in this field in particular, to extract keywords from the texts.

In reviewing the scientific literature, one finds a wide variety of terms used to designate concepts similar to what we know as automatic indexing, including such 
expressions as:

«Automated assisted indexing» «Automated indexing», «Automated support to indexing», «Automatic support to indexing», "Computer aided indexing», "Computer assistance in indexing», «Computer assisted indexing», «Computer indexing», «Computerized indexing», «Indexing by computer», «Indexing program», «Indexing software», «Machine aided indexing», «Machine indexing», «Machine-assisted indexing», «Mechanical indexing», «Mechanized indexing», «Microcomputer-based indexing», «Semi-automatic indexing», «Automatic indexing». The last of these expressions is the most often used in the literature. This terminological variety reflects three different concepts: (i) Computer programs that aid in storing the indexing terms after they have been extracted intellectually. Such systems provide help screens giving explanatory notes on the use of a term or related terms, and allowing terms to be assigned without having to key them in. They even allow any aspect of the process to be checked by on-line consultation of previously indexed documents (computer assisted indexing during storage). (ii) Systems which automatically analyse the documents, but the proposed indexing terms are then alidated and edited if necessary by a professional (semiautomatic indexing). (iii) Programs without any kind of validation, i.e., the proposed terms are stored directly as keywords or descriptors of the given document (automatic indexing) (Gil-Leiva, 1999).

The objective of the present work is to describe a bibliometric analysis of the scientific output on automatic indexing from 1956 to 2000. 


\section{Method}

The material used in the study was a set of 839 bibliographic references covering the period 1956-2000, inclusive. The references used were obtained by means of an exhaustive document search performed in two phases: the first phase, from 1994 to 1997, had already been used in an earlier study (Gil-Leiva, 1999), and de second, from 1998 to 2000, was to cover the publications of those three years.

The literature corpus was constructed in two ways: by extracting the bibliographic references from each article, book, or report that we had read in recent years, and by database searches. The databases used were LISA and ISA, as well as others not specializing in information science, but which also include relevant references - ERIC, MEDLINE, and SIGLE. We also used the PhD thesis databases Teseo, ThesNet, and Dissertation Abstracts.

All database searches were made on the "descriptor" field, so as to guarantee that the documents retrieved dealt specifically with "automatic indexing".

This material was used in a bibliometric analysis of the scientific output (distribution of authors and works, etc.), the obsolescence of the scientific literature and its dispersion, and the distribution of the literature by topic, year, source type, etc.

\section{Results}

\subsection{Scientific output by document type}

In Table 1, one can observe some of the aspects of the scientific output and document type arranged into five-year periods. Firstly, there is a notable generalized decline in the 1996-2000 period. The causes of this will not become clear until it is 
possible to analyse the data of the following five-year period. Secondly, beginning in the 1980s, there is a clear increase in the number of $\mathrm{PhD}$ theses. Lastly, we found remarkably few patents.

\subsection{Annual scientific output by topical classification}

The whole set of bibliographic references were arranged into the following thematic classification: general aspects, linguistics, automatic indexing vs manual indexing, evaluation, status of the question, automatic indexing and retrieval, automatic indexing software, and techniques and methods (Table 2).

The works with proposals of techniques and methods for automatic indexing were the most numerous (290). There were possibly two reasons for this: the complexity of undertaking this process automatically stimulated many experiments, and the lack of any corresponding methodological consensus amongst the scientific community led each researcher or group of researchers to propose different mechanisms. The second most frequent topic was that of works classified under general aspects (269), with a mix of the theory of automatic indexing, problems to be overcome, and reflections on the theme. Thirdly, there were works dealing with the relationship automatic indexing and retrieval (111).

The analysis of the thematic classification by year showed that there were two peaks in the distribution of the item general aspects, the first between the years 1969 and 1975, and the second, more marked than the other, between 1989 and 1999. The same was the case with linguistics, with one of the two peaks in the early 1970s and the other in the early 1990s. There was little change between 1969 and 2000, however, for techniques and methods, automatic indexing vs manual indexing (the debate on whether 
or not automating the process was worthwhile), and status of the question. Lastly, there was a notable peak from 1990 to 1997 corresponding to automatic indexing and retrieval.

\subsection{The scientific output of the authors}

In 1926, Alfred J. Lotka from his investigations into the frequency distribution of the scientific output of physicists and chemists, formulated a relationship between the frequency of authors and their publications.

In his article, Lotka says: "In the cases examined it is found that the number of persons making 2 contributions is about one-fourth of those making one; the number making 3 contributions is about one-ninth, etc.; the number making $x$ contributions is

about $1 / x^{2}$ of those making one; and the proportion, of all contributors, that make a single contribution, is about 60 per cent.

In other words, for every 100 authors with one article as output, there will be 25 with two articles each, about 11 with three, approximately 6 with four contributions, and so on. Lotka (1926) already formulated the general law. He found, for the two data sets he analyzed, an exponent of $2.02 \pm 0.017$, and $1.888 \pm 0.007$. Consequently, he writes " the general formula for the relation thus found to exist between the frequency of persons making $\mathrm{x}$ contributions es $x^{n} y=\operatorname{const}\left(y_{x}=c \times x^{-n}\right)$.

The methods for the calculation of the values of the constant ' $c$ ' and the slope ' $n$ ' were also defined (Pao, 1985; Nicholls, 1986). The calculation of ' $c$ ' (the value corresponding to the number of authors with a single work in Lotka's equation) requires the prior determination of the value of the slope of the distribution ' $n$ ', which in turn 
requires having decided on the number of data pairs to be used in its calculation.

While the two calculations, of ' $c$ ' and of ' $n$ ', have been resolved methodologically, the last step mentioned, the choice of the number of data pairs to use in calculating the slope, has been the object of most proposals, without any of them being accepted by the scientific community up to now. We shall determine the parameters excluding that part of the data representing the more prolific authors (at $y_{x}=1$ ).

If $x$ and $y$ follow an inverse power law, the resulting (log-log) plot will be a straight line of negative slope $n$. The value of $n$ is calculated by the least squares method:

$$
n=\frac{N \sum X Y-\sum X \sum Y}{N \sum X^{2}-\left(\sum X\right)^{2}}
$$

where

$$
\begin{aligned}
& \mathrm{N}=\text { number of data pairs considered } \\
& \mathrm{X}=\text { (decimal) logarithm of } \mathrm{x}(\mathrm{x}=\text { number of works }) \text {. } \\
& \mathrm{Y}=\text { (decimal) logarithm of }(\mathrm{y}=\text { number of authors }) \text {. }
\end{aligned}
$$

In the present case, with the data of Table 3, the slope will be the following:

$$
n=\frac{7(3.67)-(3.7 \times 9.71)}{7(2.489)-(3.7)^{2}}=-2.75
$$

The calculation of ' $c$ ' starts from Lotka's law, $y_{x}=c \times x^{-n}$. Dividing both terms by $\sum y_{x}$, the total number of authors, $y_{x} / \sum y_{x}=\left(c / \sum y_{x}\right)\left(1 / x^{n}\right)$ and writing $c / \sum y_{x}=C$, the fraction of the total sample of authors, one has $y_{x} / \sum y_{x}=C \times\left(\frac{1}{x^{n}}\right)$, and hence $\sum y_{x} / \sum y_{x}=C \sum \frac{1}{x^{n}}=1$. 
Finally $C=1 /\left(\sum 1 / x^{n}\right)$

For fractional non-negative values of $n$, the sum of the series in its general form $\sum 1 / x^{n}$ can only be approximated by a function that calculates the sum of the first $\mathrm{P}$ terms. The result, according to Pao (1985), is due to Professor David Singer:

$$
\sum_{x=1}^{\infty} 1 / x^{n}=\left[\sum_{x=1}^{P-1} \frac{1}{x^{n}}+\frac{1}{(n-1)\left(P^{n-1}\right)}+\frac{1}{2 P^{n}}+\frac{n}{24(P-1)^{n+1}}\right]
$$

For the present case, using the slope 'n'calculated before, 2.75, one has

$$
\sum_{x=1}^{\infty} 1 / x^{2.75}=\left[\sum_{x=1}^{19} \frac{1}{x^{2.75}}+\frac{1}{(2.75-1)\left(P^{1.75}\right)}+\frac{1}{2 P^{2.75}}+\frac{2.75}{24(P-1)^{3.75}}\right]=1,271
$$

and $C=1 / 1.271=0.7867$. This represents the authors with a single work in the theoretical distribution, i.e., in the expected frequencies. From this datum, and applying Lotka's law $y_{x}=c \times x^{-n}$, one completes column 9 of Table 3 .

In order to verify that the observed distribution of the productivity of the authors fits the theoretical distribution, we subjected the data to the non-parametric KolmogorovSmirnov test. To this end, we used the data in the last column of Table 3 (Dmax), obtained as the absolute value of the difference between columns 8 and 10 of the same table. The greatest value of this column (Dmax) will be taken as reference for comparison with the "critical value" (c.v.), obtained by the asymptotic formula

$$
\text { v.c. }=1,63 /\left(\sum y_{x}+\left(\sum y_{x} / 10\right)^{1 / 2}\right)^{1 / 2}
$$


For our case, we shall use a significance level of 0.01 , so that the expression will be,

$$
\text { v.c. }=1,63 /\left(883+(883 / 10)^{1 / 2}\right)^{1 / 2}=0.054
$$

with $\Sigma \mathrm{y}_{\mathrm{x}}$ being the total number of authors (in our case 883).

The data of Table 3 give a value of Dmax $=0.02$, and the critical value is 0,054 .

Since the value of the present distribution is smaller than the critical value $(0.02<$ 0.054), the null hypothesis that the data follow a Lotka distribution has to be accepted.

\subsection{The obsolescence of the scientific literature}

By "obsolescence" one understands the temporally declining utility, or use, or validity of information or measurements (Line \& Sandison, 1974).

There are two possible approaches in studying obsolescence: (i) a diachronous study, which takes a certain moment in time as the starting point, and follows the impact that a body of literature has on the surrounding science as measured by the citations it receives in the years following publication; and (ii) a synchronous study, which analyses the antiquity of the references that the body of literature has cited and on which its own contribution is based.

Burton and Kebler (1960) introduced the concept of "half-life" into the field of information science, finding that the half-life of the references in the journals of various sciences depends on the topical area concerned. Beltram C. Brookes (1970) established the mathematical law describing the temporal loss of utility of a set of documents.

The half-life $h$ is an indicator of obsolescence, and represents the age at which the utility (number of references or citations) has fallen by a half. We shall start with Brookes' formulation $a^{h}=0,5$ to calculate the obsolescence of the "automatic 
indexing" literature.

The references used are from 1952 to 2000 . Table 4 gives the references from 1962, leaving out the 13 references published earlier. The year with most references is 1969, with 49, followed by 1990 with 47. Separated into decades, the most productive was clearly that of the 1990s.

From Brookes' equation, with appropriate operations, one has:

$$
a=e^{\left(\frac{\ln 0,5}{h}\right)}
$$

where $\mathrm{a}=$ annual aging factor.

Table 4 shows that the half-life, $h$, the age at which the utility is reduced by half, is between 14 and 15 years. For its exact calculation, we interpolate between columns 2 and 5 of Table 4:

$$
\frac{0,509-0,489}{14-15}=\frac{0,509-0,500}{14-h}=14,47 \text { years }=h
$$

Substituting this value in the equation for $a=e^{\left(\frac{\ln 0,5}{h}\right)}$, one has:

$$
a=e^{\left(\frac{\ln 0,5}{14,78}\right)}=0,95
$$

This is an annual aging factor of $95 \%$, or an annual loss of currency of $5 \%$.

Considering either the value calculated for $h$ or the annual aging factor, one observes that this literature is very stable from the perspective of its use, since a half-life of 14.47 years means that it takes about 15 years for the utility of this literature to be reduced by $50 \%$. 


\subsection{Dispersion of the scientific literature (Bradford's law)}

One of the milestones in the development of bibliometrics and information science was the evidence for the regularity in the distribution of scientific journals, known as Bradford's law (Bradford, 1934, 1948).

Bradford found that, on dividing the journals into three zones, each with the same number of articles, that the number of journals in each zone grew geometrically. Also, the distribution of journals according to their productivity presented a different model of concentration and dispersion when it was represented as a statistical distribution, with a larger group forming a long tail of less productive journals.

Evidently the implications of these findings went beyond the mere description of the dispersion of the scientific literature in journals. Aspects as wide-reaching as the rinciples by which the scientific community functions as a stable integrated system, or the universality of the application of Bradford's formulation derive from this regularity.

In presenting the formulation of his empirical law of the literature in scientific journals, Bradford included a graph as illustration. Along the x-axis, he placed the journals $1,2,3, \ldots, \mathrm{r}$ in decreasing order of productivity of relevant works on a given topic, using a logarithmic scale. The y-axis represented the accumulated total of publications $\mathrm{R}(\mathrm{r})$. The resulting semi-log plot began with a curve which, beyond a critical point, became a straight line.

Subsequently, Leimkuhler (1967), Brookes (1969), and Egghe (1990) have given mathematical expressions for Bradford's law. Egghe's method is based on the earlier 
formulation of Leimkuhler:

$$
R(r)=a \log _{e}(1+b r)
$$

Using the following notation:

$\mathrm{r}_{0}=$ number of journals in Bradford's first group

$\mathrm{y}_{0}=$ number of articles in each group (all groups are of the same size)

$\mathrm{k}=$ Bradford's multiplier

$\mathrm{Y}_{\mathrm{m}}=$ number of articles in the most productive journal (rank 1)

$\mathrm{R}(\mathrm{r})=$ cumulative number of articles produced by the journals of rank $1,2,3, \ldots, \mathrm{r}$

$\mathrm{a}$ and $\mathrm{b}=$ constants that appear in Leimkuhler's formulation.

Egghe showed that: $a=\frac{y_{0}}{\log _{e} k}$, for the present case $a=\frac{132}{1,91}=110.8$.

$b=\frac{k-1}{r_{0}}$, for the present case $b=\frac{2,29}{3,60}=0,64$.

$\mathrm{P}=$ number of Bradford groups, and also determined $\mathrm{k}$ :

$$
k=\left(e^{\gamma} \times Y_{m}\right)^{1 / P}
$$

$\gamma=$ Euler's number $=0,5772 ; \mathrm{e}^{\gamma}=1,781$

$$
k=\left(1,781 \times Y_{m}\right)^{1 / P}
$$


$\mathrm{A}=$ number of articles in the literature

$$
y_{0}=\frac{A}{P}
$$

$\mathrm{T}=$ total number of journals.

$$
r_{0}=\frac{T(k-1)}{k^{P}-1}
$$

To calculate $r_{1}, r_{2}, r_{3}, \ldots .$. ., one uses the exact value for $r_{0}$ and, with rounding off, that of K.

$$
r_{0} \times 1=r_{0} ; r_{0} \times k=r_{1} ; r_{0} \times k^{2}=r_{2} ; r_{0} \times k^{3}=r_{3}
$$

With the data of Table 5, and using Egghe's procedure, we obtain the following results:

We chose $\mathrm{P}=4$ as being the ideal number of zones for the distribution, so that

$$
k=(1,781 \times 66)^{1 / 4}=3,29 \text { and } r_{0}=\frac{183 \times 2,29}{117,2-1}=3,6
$$

\section{Bradford's distribution}

\begin{tabular}{|c|c|c|c|}
\hline Zones & Number of journals & Number of articles & K \\
\hline Core & 4 & 170 & -- \\
\hline Zone 1 & 12 & 105 & 3.00 \\
\hline Zone 2 & 39 & 113 & 3.25 \\
\hline Zone 3 & 128 & 139 & 3.28 \\
\hline
\end{tabular}

The similarity of the different values of $\mathrm{k}$, and between these and the calculated 
Bradford multiplier (3.29), clearly show that the distribution fits a four-zone Bradford law.

The equation for the Bradford curve in the present case is:

$$
R(r)=110,8 \log _{e}(1+0,64 r) .
$$

\section{Conclusions}

Automating the task of indexing has been a theme of constant interest for researchers from the 1950s until the present day, as this study of more than 800 research works published between 1956 and 2000 has shown. In the last five-year period, however, there has been a considerable decline in scientific output with respect to the previous five-year periods, perhaps because of the lag in updating some of the databases. The scientific output corresponding to "PhD theses", however, has not fallen.

With respect to scientific output by topic, the most productive was that of "techniques and methods", followed by "general aspects". There were certain variations observed within the topics in some cases.

The author distribution by productivity was examined following the approach of Lee Pao (1985). The result of applying the Kolmogorov-Smirnov goodness-of-fit test was that it did fit a Lotka distribution $($ Dmax $=0.02$ vs critical value $=0.054)$.

We applied the concept of half-life to calculate the obsolescence of the scientific literature, finding an annual aging factor of 95\%, i.e., a 5\% annual loss of currency. In terms of obsolescence, this percentage means that this literature has a low level of aging, with a half-life close to 15 years (meaning that the use of this literature is reduced by $50 \%$ every 15 years). 
Finally, the dispersion of the bibliographic corpus that was analysed was found to be low, given the number of journals in which the articles were published. There were 527 articles published in 183 journals. The most productive journal published 66 articles, while 117 journals published only a single article. There therefore exists a concentration of articles in a small number of journals. The journals of the core and of zone 1 (16 journals altogether) accounted for more than 50\% of the articles (275).

\section{References.}

Anderson, J. D., \& Pérez-Carballo, J. (2001). The nature of indexing: how humans and machines analyze messages and texts for retrieval. Part I: Research, and the nature of human indexing. Information Processing \& Management,37, 231-254.

Anderson, J. D., \& Pérez-Carballo, J. (2001). The nature of indexing: how humans and machines analyze messages and texts for retrieval. Part II: Machine indexing, and the allocation of human versus machine effort. Information Processing \& Management,37, 255-277.

Bradford, S. C. (1934). Sources of information on specific subjects. Engineering, 23(3), 85-88.

Bradford, S. C. (1948). Documentation. London: Crosby, Lockwood Sons Ltd.

Brookes, B. C. (1969). Bradford's law and the bibliography of science. Nature, 22(5523), 953-956.

Brookes, B. C. (1970). Obsolescence of special library periodicals: sampling errors and utility contours. Journal of the American Society for Information Science, 21(5), 320-329.

Burton, E., \& Kebler, R. W. (1960). The "Half-life" of some scientific and technical literatures. American Documentation, 11(1), 18-22.

Egghe, L. (1990). Applications of the theory of Bradford's law to the calculation of Leimkuhler's law and to the completion of bibliographies. Journal of the American Society for Information Science, 41(7), 469-492.

Gil-Leiva, I. (1999). La automatización de la indización. Gijón, Spain: Trea.

Leimkuhler, F. F. (1967). The Bradford distribution. Journal of Documentation, 23, 197-207.

Line, M. B., \& Sandison, A. (1974). Obsolescence and changes in the use of the literature with time. Journal of Documentation, 30(3), 283-350.

Lotka, A. J. (1926). The frequency distribution of scienctific productivity. Journal of the Washington Academy of Sciences, 16(12), 317-323.

Nicholls, P. T. (1986). Empirical validation of Lotka's law. Information Processing \& Management, 22(5), 417-419.

Pao, M. L. (1985). Lotka’s law: a testing procedure. Information Processing \& Management, 21(4), 305320. 
Information Processing \& Management, 2004, vol. 40, n 2, p. 365-377.

Table 1

Scientific production by document type

\begin{tabular}{lcccccccc}
\hline Years & Journal articles & Books and book chapters & Congresses & Theses & Reports & Memoires & Patents & Total \\
\hline $1996-2000$ & 81 & 4 & 12 & 14 & 1 & 0 & 0 & 112 \\
$1991-1995$ & 118 & 12 & 32 & 11 & 7 & 0 & 1 & 181 \\
$1986-1990$ & 76 & 17 & 25 & 14 & 0 & 2 & 1 & 135 \\
$1981-1985$ & 48 & 14 & 5 & 12 & 2 & 1 & 0 & 82 \\
$1976-1980$ & 48 & 10 & 7 & 3 & 2 & 1 & 0 & 71 \\
$1971-1975$ & 76 & 18 & 9 & 2 & 4 & 0 & 0 & 109 \\
$1966-1970$ & 64 & 31 & 18 & 1 & 4 & 0 & 0 & 118 \\
$1961-1965$ & 12 & 5 & 4 & 1 & 1 & 0 & 0 & 23 \\
$1952-1960$ & 4 & 3 & 0 & 0 & 1 & 0 & 0 & 8 \\
Total & 527 & 114 & 112 & 58 & 22 & 4 & 2 & 839 \\
\hline
\end{tabular}


Table 2

Annual scientific production by topical classification

\begin{tabular}{|c|c|c|c|c|c|c|c|c|c|}
\hline Year & $\begin{array}{c}\text { General } \\
\text { aspects }\end{array}$ & Linguistics & $\begin{array}{l}\text { Automatic } \\
\text { indexing } \\
\text { vs manual } \\
\text { indexing }\end{array}$ & Evaluation & $\begin{array}{l}\text { Status of } \\
\text { the } \\
\text { question }\end{array}$ & $\begin{array}{l}\text { Automatic } \\
\text { indexing and } \\
\text { retrieval }\end{array}$ & $\begin{array}{l}\text { Automatic } \\
\text { indexing } \\
\text { software }\end{array}$ & $\begin{array}{l}\text { Techniques } \\
\text { and methods }\end{array}$ & Total \\
\hline 2000 & 1 & 0 & 0 & 0 & 1 & 1 & 2 & 8 & 13 \\
\hline 1999 & 11 & 0 & 1 & 0 & 1 & 2 & 0 & 4 & 19 \\
\hline 1998 & 12 & 1 & 0 & 1 & 0 & 4 & 0 & 9 & 27 \\
\hline 1997 & 9 & 2 & 1 & 0 & 0 & 9 & 0 & 5 & 26 \\
\hline 1996 & 12 & 1 & 1 & 1 & 2 & 3 & 0 & 7 & 27 \\
\hline 1995 & 6 & 0 & 0 & 2 & 0 & 14 & 1 & 8 & 31 \\
\hline 1994 & 13 & 2 & 0 & 1 & 1 & 5 & 1 & 12 & 35 \\
\hline 1993 & 13 & 3 & 0 & 0 & 2 & 4 & 1 & 16 & 39 \\
\hline 1992 & 9 & 6 & 3 & 3 & 2 & 3 & 2 & 11 & 39 \\
\hline 1991 & 12 & 8 & 0 & 0 & 1 & 1 & 0 & 15 & 37 \\
\hline 1990 & 11 & 9 & 1 & 1 & 2 & 8 & 3 & 12 & 47 \\
\hline 1989 & 10 & 1 & 1 & 0 & 0 & 4 & 1 & 5 & 22 \\
\hline 1988 & 7 & 3 & 2 & 1 & 1 & 4 & 3 & 10 & 31 \\
\hline 1987 & 4 & 0 & 0 & 0 & 0 & 3 & 3 & 9 & 19 \\
\hline 1986 & 2 & 2 & 0 & 1 & 0 & 3 & 1 & 7 & 16 \\
\hline 1985 & 2 & 1 & 0 & 1 & 1 & 0 & 1 & 8 & 14 \\
\hline 1984 & 7 & 1 & 1 & 1 & 0 & 2 & 0 & 12 & 24 \\
\hline 1983 & 3 & 1 & 0 & 0 & 0 & 0 & 1 & 12 & 17 \\
\hline 1982 & 4 & 2 & 0 & 0 & 1 & 0 & 0 & 4 & 11 \\
\hline 1981 & 3 & 3 & 0 & 0 & 0 & 1 & 2 & 7 & 16 \\
\hline 1980 & 4 & 1 & 0 & 0 & 0 & 0 & 0 & 9 & 14 \\
\hline 1979 & 3 & 2 & 1 & 0 & 0 & 1 & 0 & 5 & 12 \\
\hline 1978 & 6 & 0 & 0 & 2 & 0 & 1 & 0 & 8 & 17 \\
\hline 1977 & 4 & 1 & 2 & 0 & 2 & 0 & 0 & 8 & 17 \\
\hline 1976 & 2 & 2 & 0 & 1 & 0 & 0 & 0 & 6 & 11 \\
\hline 1975 & 8 & 0 & 0 & 2 & 2 & 1 & 0 & 8 & 21 \\
\hline 1974 & 9 & 0 & 0 & 0 & 3 & 2 & 0 & 5 & 19 \\
\hline 1973 & 6 & 1 & 0 & 1 & 2 & 1 & 0 & 9 & 20 \\
\hline 1972 & 4 & 4 & 1 & 0 & 2 & 4 & 0 & 6 & 21 \\
\hline 1971 & 12 & 1 & 0 & 0 & 1 & 6 & 1 & 7 & 28 \\
\hline 1970 & 11 & 4 & 0 & 4 & 0 & 9 & 0 & 16 & 44 \\
\hline 1969 & 21 & 2 & 3 & 2 & 1 & 9 & 0 & 11 & 49 \\
\hline 1968 & 6 & 1 & 0 & 2 & 0 & 1 & 0 & 2 & 12 \\
\hline 1967 & 5 & 0 & 0 & 0 & 0 & 2 & 0 & 0 & 7 \\
\hline 1966 & 3 & 1 & 0 & 0 & 1 & 0 & 0 & 1 & 6 \\
\hline 1965 & 2 & 0 & 0 & 0 & 1 & 1 & 0 & 3 & 7 \\
\hline 1964 & 2 & 0 & 0 & 1 & 0 & 0 & 0 & 1 & 4 \\
\hline 1963 & 3 & 0 & 0 & 0 & 1 & 0 & 0 & 1 & 5 \\
\hline 1962 & 2 & 0 & 0 & 0 & 0 & 0 & 0 & 0 & 2 \\
\hline 1961 & 2 & 1 & 0 & 0 & 2 & 0 & 0 & 0 & 5 \\
\hline 1960 & 0 & 0 & 0 & 0 & 0 & 1 & 0 & 0 & 1 \\
\hline 1959 & 0 & 0 & 0 & 0 & 0 & 0 & 0 & 1 & 1 \\
\hline 1958 & 2 & 0 & 0 & 0 & 0 & 1 & 0 & 1 & 4 \\
\hline 1957 & 0 & 0 & 0 & 0 & 0 & 0 & 0 & 1 & 1 \\
\hline 1956 & 1 & 0 & 0 & 0 & 0 & 0 & 0 & 0 & 1 \\
\hline Total & 269 & 67 & 18 & 28 & 33 & 111 & 23 & 290 & 839 \\
\hline
\end{tabular}


Table 3

Author productivity

\begin{tabular}{lllllllllll}
\hline $\mathrm{X}$ & $\mathrm{Y}_{\mathrm{x}}$ & $\mathrm{X}=\lg \mathrm{x}$ & $\mathrm{Y}=\lg \mathrm{y}$ & $\mathrm{XX}$ & $\mathrm{XY}$ & $\mathrm{Yx} / \sum \mathrm{Yx}$ & $\sum\left(\mathrm{Yx} / \sum \mathrm{Yx}\right)$ & fe & $\sum \mathrm{fe}$ & $\mathrm{D}$ \\
\hline 1 & 703 & 0 & 2,8469 & 0 & 0 & 0,7961 & 0,7961 & 0,7867 & 0,7867 & 0,0094 \\
2 & 112 & 0,3010 & 2,0492 & 0,0906 & 0,6168 & 0,1268 & 0,9229 & 0,1169 & 0,9036 & 0,0193 \\
3 & 35 & 0,4771 & 1,5440 & 0,2276 & 0,7366 & 0,0396 & 0,9625 & 0,0383 & 0,9419 & $0,0206 *$ \\
4 & 11 & 0,6020 & 1,0410 & 0,3624 & 0,6267 & 0.0124 & 0,9749 & 0,0173 & 0,9592 & 0,0157 \\
5 & 8 & 0,6989 & 0,9030 & 0,4885 & 0,6311 & 0,0090 & 0,9839 & 0,0094 & 0,9686 & 0,0153 \\
6 & 7 & 0,7781 & 0,8450 & 0,6055 & 0,6575 & 0,0079 & 0,9918 & 0,0057 & 0,9743 & 0,0175 \\
7 & 3 & 0,8450 & 0,4771 & 0,7141 & 0,4031 & 0,0034 & 0,9952 & 0,0037 & 0,9780 & 0,0172 \\
9 & 1 & 0,9542 & 0 & 0,9105 & 0 & 0,0011 & 0,9963 & 0,0017 & 0,9797 & 0,0166 \\
10 & 1 & 1,0000 & 0 & 1,0000 & 0 & 0,0011 & 0,9974 & 0,0013 & 0,9810 & 0,0164 \\
14 & 1 & 1,1461 & 0 & 1,3136 & 0 & 0,0011 & 0,9985 & 0,0005 & 0,9815 & 0,0170 \\
34 & 1 & 1,5314 & 0 & 2,3454 & 0 & 0,0011 & 0,9996 & 0,0004 & 0,9819 & 0,0177 \\
$\sum$ & 883 & 8,33 & 9,71 & 8,03 & $3,67 * *$ & & & & &
\end{tabular}

**Totals excluding the data $y_{x}=1$.

$\mathrm{x}=$ number of works

$\mathrm{y}=$ number of authors

$\mathrm{Yx} / \sum \mathrm{Yx}$ = frequency of authors with a single work, with two, with three, etc. (the frequencies observed in the distribution of authors on automatic indexing).

$\sum\left(\mathrm{Yx} / \sum \mathrm{Yx}\right)=$ cumulative frequency of authors with one, two, etc. works.

$\mathrm{fe}=$ expected frequencies, calculated by Lotka's formula (the value of the first cell corresponds to the value of ' $C$ ').

$\sum \mathrm{fe}=$ cumulative expected frequencies.

$\mathrm{D}=$ Dmax $=$ differences between the columns of the observed and expected cumulative frequencies. 
Table4

The obsolescence of the scientific literature.

\begin{tabular}{|c|c|c|c|c|}
\hline Years & Age, $\mathrm{t}$ & Refs/year & Acumulated Refs & Utility \\
\hline 2000 & 0 & 13 & 839 & 1.000 \\
\hline 1999 & 1 & 19 & 826 & 0,984 \\
\hline 1998 & 2 & 27 & 807 & 0,961 \\
\hline 1997 & 3 & 26 & 780 & 0,929 \\
\hline 1996 & 4 & 27 & 754 & 0,898 \\
\hline 1995 & 5 & 31 & 727 & 0,866 \\
\hline 1994 & 6 & 35 & 696 & 0,829 \\
\hline 1993 & 7 & 39 & 661 & 0,788 \\
\hline 1992 & 8 & 39 & 622 & 0,741 \\
\hline 1991 & 9 & 37 & 583 & 0,695 \\
\hline 1990 & 10 & 47 & 546 & 0,650 \\
\hline 1989 & 11 & 22 & 499 & 0,595 \\
\hline 1988 & 12 & 31 & 477 & 0,568 \\
\hline 1987 & 13 & 19 & 446 & 0,531 \\
\hline 1986 & 14 & 16 & 427 & 0,509 \\
\hline 1985 & 15 & 14 & 411 & 0,489 \\
\hline 1984 & 16 & 24 & 397 & 0,473 \\
\hline 1983 & 17 & 17 & 373 & 0,444 \\
\hline 1982 & 18 & 11 & 356 & 0,424 \\
\hline 1981 & 19 & 16 & 345 & 0,411 \\
\hline 1980 & 20 & 14 & 329 & 0,392 \\
\hline 1979 & 21 & 12 & 315 & 0,375 \\
\hline 1978 & 22 & 17 & 303 & 0,360 \\
\hline 1977 & 23 & 17 & 286 & 0,340 \\
\hline 1976 & 24 & 11 & 269 & 0,319 \\
\hline 1975 & 25 & 21 & 258 & 0,307 \\
\hline 1974 & 26 & 19 & 237 & 0,282 \\
\hline 1973 & 27 & 20 & 218 & 0,258 \\
\hline 1972 & 28 & 21 & 198 & 0,234 \\
\hline 1971 & 29 & 28 & 177 & 0,210 \\
\hline 1970 & 30 & 44 & 149 & 0,177 \\
\hline 1969 & 31 & 49 & 105 & 0,124 \\
\hline 1968 & 32 & 12 & 56 & 0,066 \\
\hline 1967 & 33 & 7 & 44 & 0,052 \\
\hline 1966 & 34 & 6 & 37 & 0,043 \\
\hline 1965 & 35 & 7 & 31 & 0,037 \\
\hline 1964 & 36 & 4 & 24 & 0,028 \\
\hline 1963 & 37 & 5 & 20 & 0,024 \\
\hline 1962 & 38 & 2 & 15 & 0,018 \\
\hline
\end{tabular}


Information Processing \& Management, 2004, vol. 40, n 2, p. 365-377.

Table5

The dispersion of the scientific literature

\begin{tabular}{ccccc}
\hline No. of journals & No. of articles & Cumulative journals & Cumulative articles & Ln (cum. Jour.) \\
\hline 1 & 66 & 1 & 66 & 0 \\
1 & 40 & 2 & 106 & 0,6931 \\
1 & 36 & 3 & 142 & 1,0986 \\
1 & 28 & 4 & 170 & 1,3862 \\
1 & 21 & 5 & 191 & 1,6094 \\
1 & 18 & 6 & 209 & 1,7917 \\
1 & 10 & 7 & 219 & 1,9459 \\
1 & 8 & 8 & 227 & 2,0794 \\
2 & 7 & 10 & 241 & 2,3025 \\
4 & 6 & 14 & 265 & 2,6390 \\
5 & 5 & 19 & 290 & 2,9444 \\
6 & 4 & 25 & 314 & 3,2188 \\
14 & 3 & 39 & 356 & 3,6635 \\
27 & 2 & 66 & 410 & 4,1896 \\
117 & 1 & 183 & 527 & 5,2094 \\
\hline
\end{tabular}

